\title{
Numerical Simulation of Bidimensional Incompressible Flows Between Moving Surfaces
}

C.A. PEDROSO 1 , A.L. de BORTOLI 2 , Department of Pure and Applied Mathematics, Federal University of Rio Grande do Sul, 90501-900 Porto Alegre, RS, Brazil.

\begin{abstract}
The goal of the present work is the numerical simulation of flows between moving surfaces. The method is based on the finite differences explicit Runge-Kutta multi-stage scheme with central spatial discretization and for second order time appoximations. Tests are carried out for parallel plates (Couette) flow and moving cylindrical surfaces for newtonian and non-newtonian (journal bearings) fluids, with and without temperature variations and the results showed to compare well with analytical, numerical or experimental data found in the literature.
\end{abstract}

\section{Introduction}

One of the most common applications of viscous fluids is the flow between moving surfaces. It is usefull to introduce a fluid between surfaces, such as a viscoelastic fluid, to reduce the friction force and to minimize damage to them, which can be done in a variety of ways. Such fluids have usually a non newtonian behaviour, meaning that there exists a non linear relation between tension and deformation.

The numerical analysis of non newtonian flows is not a trivial task, requiring experience with numerical methods. In this way, before developing a numerical code for non-newtonian flows it is advised to calibrate it to the newtonian case. Common problems such as a Couette flow between parallel [8] plates or rotanting concentric/excentric cylinders with and without temperature changes can be analysed.

One of the methods employed for the numerical solution [1] of fluid flows is the method of finite differences, because of its simplicity, low cost and ability in well representing such flows. This method is not new and many other alternatives could be employed, such as finite elements, finite volume, boundary elements and spectral methods. Each of these methods has its own advantages and disadvantages, which will not be discussed here.

The method follows the central differences spatial discretization based on the explicit Runge-Kutta time-stepping scheme. Although for implicit methods bigger time-steps could be employed, it is preferable to use explicit ones like Successive

\footnotetext{
${ }^{1}$ clem@mat.ufrgs.br

2 dbortoli@mat.ufrgs.br
} 
Relaxation and Runge-Kutta, which are simple to implement, and admit parallel and vectorial code structures. It is advised to employ the simplified Runge-Kutta time-stepping scheme.

Numerical tests are carried out for parallel plates (Couette) flow between concentric and excentric cylinders, for newtonian and non newtonian fluids, taking into account temperature variations. Comparisons were made for velocity profiles, temperature distribution and streamlines. The non newtonian model employed is based on the Convected Maxwell model, which applies for small non linear relations between tension and deformation. The complete set of governing equations for these complex flows are presented in the next section.

\section{Governing Equations}

The complete set of governing equations for general fluid flows is formed by the mass conservation, momentum and energy equations. Such set for bidimensional incompressible flows can be written in the general adimensionalized form as follows [2].

Momentum equations

$\mathrm{X}$ direction

$$
\frac{\partial u}{\partial t}+\frac{\partial u u}{\partial x}+\frac{\partial u v}{\partial y}=-\frac{\partial p}{\partial x}+\frac{1}{R_{e}}\left(\frac{\partial \tau_{x x}}{\partial x}+\frac{\partial \tau_{x y}}{\partial y}\right),
$$

$\mathrm{Y}$ direction

$$
\frac{\partial v}{\partial t}+\frac{\partial u v}{\partial x}+\frac{\partial v v}{\partial y}=-\frac{\partial p}{\partial y}+\frac{1}{R_{e}}\left(\frac{\partial \tau_{x y}}{\partial x}+\frac{\partial \tau_{y y}}{\partial y}\right)-\frac{R_{a} T}{P_{r} R_{e}{ }^{2}},
$$

Poisson's pressure equation

$$
\nabla^{2} p=\left[\frac{\partial^{2}(u u)}{\partial x^{2}}+2 \frac{\partial^{2}(u v)}{\partial x \partial y}+\frac{\partial^{2}(v v)}{\partial y^{2}}\right]+\frac{1}{R_{e}}\left(\frac{\partial \tau_{x x}}{\partial x}+2 \frac{\partial \tau_{x y}}{\partial y \partial x}+\frac{\partial \tau_{y y}}{\partial y}\right)+\frac{\partial D}{\partial t},
$$

Energy conservation

$$
\frac{D T}{d t}=\frac{1}{R_{e} P_{r}}\left(\frac{\partial^{2} T}{\partial x^{2}}+\frac{\partial^{2} T}{\partial y^{2}}\right)+\frac{E_{c}}{R_{e}}\left(\frac{\partial u \tau_{x y}}{\partial x}+\frac{\partial u \tau_{x y}}{\partial y}+\frac{\partial v \tau_{x y}}{\partial x}+\frac{\partial v \tau_{y y}}{\partial y}\right),
$$

where $u$ and $v$ are the velocity vector components, $T$ the temperature, $D T / d t$ the convective terms derivative, $p$ the pressure and $\tau_{i, j}$ the components of stress tensor. Such equations are valid for newtonian as well as non newtonian fluids.

A Boussinesq approximation [2], [6] was employed which relates the velocitytemperature coupling, being usefull for small temperature differences and for very small fluid compressibility. For gases and water the hypothesis of Stokes apply and one obtains the well known Navier-Stokes equations. However, the majority of fluids found in nature has a non-newtonian behaviour, which can be modeled by a 
variety of models. One of the simple and efficient one is the Convected Maxwell model [9], which can be written as

$$
\tau+\lambda \frac{\partial \tau}{\partial t}=2 \eta \frac{\partial \gamma}{\partial t}
$$

here $\tau$ represent the tensions, $\lambda$ the relaxation time, $\eta$ the dynamical viscosity, $\dot{\tau}$ the extra tension and $\dot{\gamma}$ the deformation rate.

The idea of this model is the junction of a dashpot and a spring combined in series. After the necessary adimensionalizations we obtain the following form for tension equations [9]

$$
\begin{gathered}
\tau_{x x}+D_{e}\left(u \frac{\partial \tau_{x x}}{\partial x}+v \frac{\partial \tau_{x x}}{\partial y}-2 \frac{\partial u}{\partial x} \tau_{x x}-2 \frac{\partial u}{\partial y} \tau_{x y}\right)=2 \frac{\partial u}{\partial x} \\
\tau_{x y}+D_{e}\left(u \frac{\partial \tau_{x y}}{\partial x}+v \frac{\partial \tau_{x y}}{\partial y}-\frac{\partial u}{\partial y} \tau_{y y}-\frac{\partial u}{\partial x} \tau_{x x}\right)=\left(\frac{\partial u}{\partial y}+\frac{\partial v}{\partial x}\right) \\
\tau_{y y}+D_{e}\left(u \frac{\partial \tau_{y y}}{\partial x}+v \frac{\partial \tau_{y y}}{\partial y}-2 \frac{\partial v}{\partial y} \tau_{y y}-2 \frac{\partial u}{\partial x} \tau_{x y}\right)=2 \frac{\partial v}{\partial y}
\end{gathered}
$$

where $D_{e}$ is the Deborah number $(\lambda V / L)$, for which $\lambda$ is equal to zero for the Newtonian case. This number corresponds to the rate between the temporal and process characteristics or, in other words, it is responsible for the "fluid memory".

\section{Description of Numerical Method}

The finite differences three-stages Runge-Kutta scheme with central spatial discretization is employed, because of its implementation simplicity and efficiency when analysisng these fluid flows [1]. For solving pressure equation a Successive Relaxation for Gauss-Seidel scheme was employed; such method can be understood as a Runge-Kutta of one time-step.

A good mesh generation is very important when solving fluid problems such as between excentric cylinders. Appropriate concentration must be obtained near wall surfaces in order to well represent variable gradients. Significant computational time could be reduced when using appropriate grids.

The set of governing equations must be transformed into a generalized coordinate system [10]. Following the transformation $(\mathrm{x}, \mathrm{y}, \mathrm{t})$ to $(\xi, \eta, t)$ a set of metrics is obtained. Based on the following transformation the momentum in $\mathrm{X}$ direction results in the following form

$$
\begin{aligned}
& \frac{\partial u}{\partial t}+\frac{\partial u u}{\partial \xi} \xi_{x}+\frac{\partial u u}{\partial \eta} \eta_{x}+\frac{\partial v u}{\partial \xi} \xi_{y}+\frac{\partial v u}{\partial \eta} \eta_{y}=-\left(\frac{\partial p}{\partial \xi} \xi_{x}+\frac{\partial p}{\partial \eta} \eta_{x}\right)+ \\
& +\frac{1}{R_{e}}\left(\frac{\partial \tau_{x x}}{\partial \xi} \xi_{x}+\frac{\partial \tau_{x x}}{\partial \eta} \eta_{x}+\frac{\partial \tau_{x y}}{\partial \xi} \xi_{y}+\frac{\partial \tau_{x y}}{\partial \eta} \eta_{y}\right)
\end{aligned}
$$

where $\xi_{x}, \eta_{x}, \xi_{y}$ and $\eta_{y}$ are the metrics of coordinates transformation given, for example, by 


$$
\xi_{x}=J y_{\eta}
$$

Here, J represents the Jacobian (areas relation). The other equations are written in a similar form.

All terms of the complete set of governing equations are approximated based on central differences [1], [3] for convective as well as diffusive terms based on the Runge-Kutta coefficients for seccond order time integration with convergence criterion based on pressure or temperature of order $\epsilon<0.0001$. The developed code is based on the following algorithm:

1. initialize variables;

2. obtain metrics of coordinates transformation, when necessary;

3. apply initial and boundary conditions;

4. calculate tension components;

5. obtain velocity vector components;

6. evaluate the pressure;

7. obtain the temperature;

8. return to item 4 until convergence or stage required is reached.

\section{Numerical Results}

In the following, numerical results for parallel plate and rotating excentric cylinders are presented and compared with ones found in the literature. Streamlines, isotherms and velocity profiles are used to verify and to calibrate the code.

First results were obtained for Couette flow for various pressure gradients (-3, $-2,-1,0,1,2,3)$. The adimensional pressure is defined as follows

$$
P=-\frac{h^{2}}{2 \mu U} \frac{d p}{d x}
$$

where $\mathrm{h}$ is the plates distance, $\mu$ the kinematic fluid viscosity, $\mathrm{U}$ the flow velocity and $d p / d x$ the dimensional pressure gradient. The analytical solution gives the following velocity distribution

$$
u=\frac{y}{h} U+P \frac{y}{h}\left(1-\frac{y}{h}\right),
$$

where $\mathrm{y}$ is the distance from the fixed plate in the direction to the moving plate.

Fig. 1 presents the velocity profiles for various adimensional pressure gradients. Such results are in good agreement, for all constant pressure lines, with the ones obtained by Schlichting [8]. 


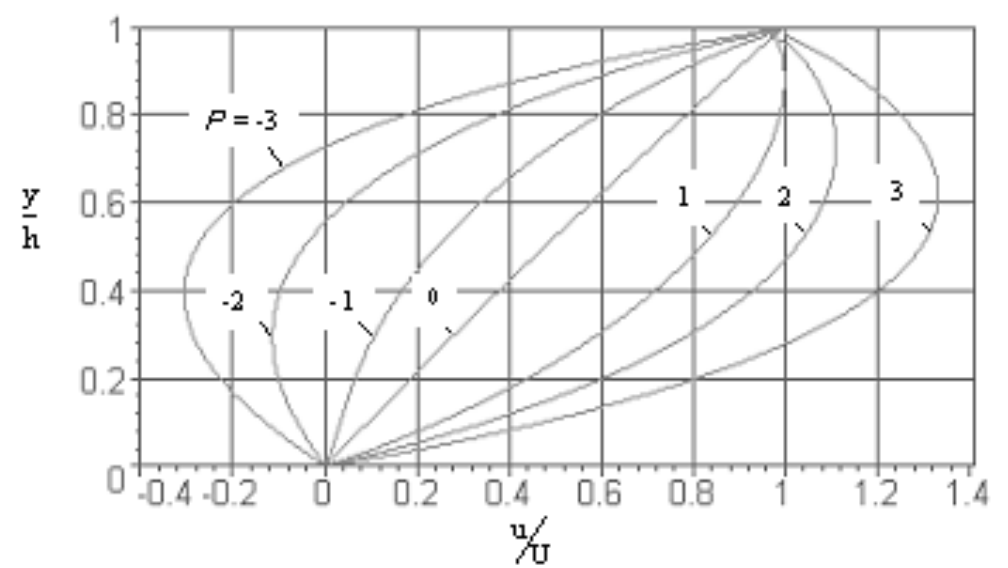

Fig. 1 Velocity profiles for various adimensional pressure gradients

Fig. 2 displays the velocity profile comparison for the product $P_{r} E_{c}=8$ among the plates. The results are in good agreement as expected after obtaining good velocity profiles agreement.

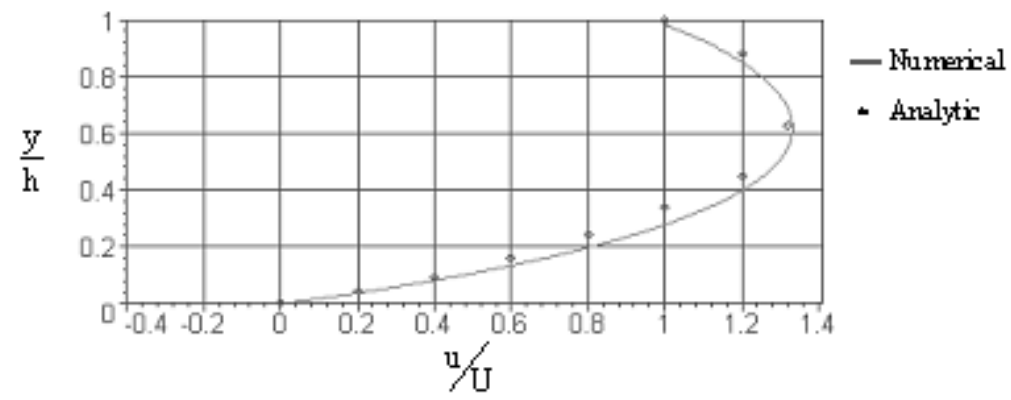

Fig. 2 Comparison of the velocity profiles for $P=0.8$ with the results from Schlichting [8]

In the following, a temperature distribution is presented and compared. The analytical solution in adimensional form is given by

$$
\frac{T-T_{0}}{T_{1}-T_{0}}=\frac{y}{h}+P_{r} E_{c} \frac{y}{h}\left(1-\frac{y}{h}\right)
$$

where $T_{0}$ is the fixed wall temperature and $T_{1}$ the moving plate temperature. Fig. 3 shows the temperature profile for different $P_{r} E_{c}$ numbers, from 0 to $8(0,1,2,4,8)$. The results show good agreement for higher $P_{r} E_{c}$ but not between these adimensional variables. This difficulty can be associated to the strong velocity-pressure coupling in the numerical technique employed, while the coupling between pressuretemperature or velocity-temperature is weak. In any case, such results are acceptable because of the small differences presented. 


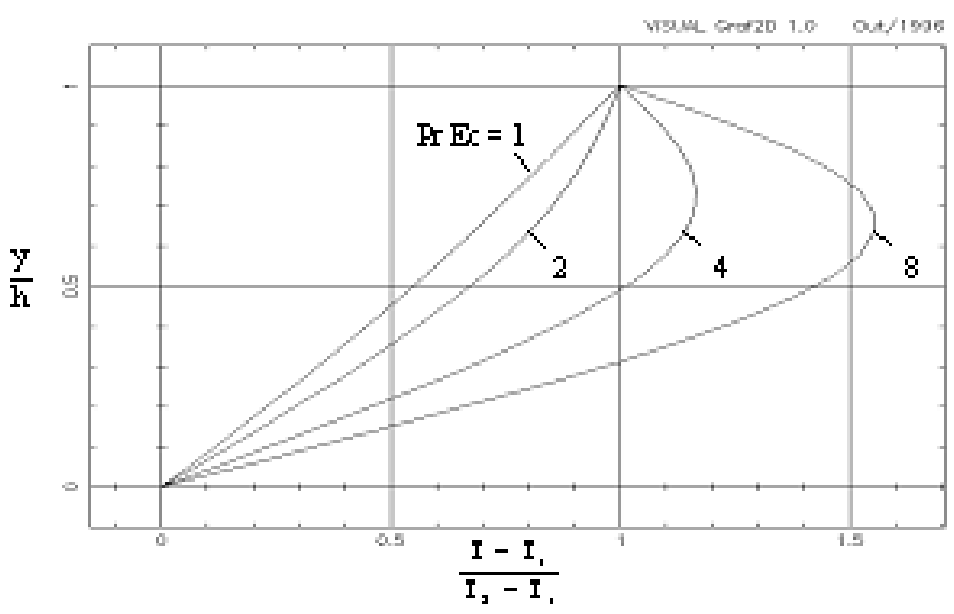

Fig. 3 Temperature distribution for Couette flow for various $P_{r} E_{c}$ products

After these comparisons for a simple flow situation, numerical results for the flow between concentric and excentric cylinders is presented based on the boundary fitted coordinates system. First results were obtained for newtonian flow between concentric cylinders, whose mesh is presented in Fig. 4. Such results were obtained for $P_{r}=0.71$ and $R_{e}=300$ for external diameter 2.6 times the internal one and angular velocity 1, as can be seen in Fig. 5. The comparison with experimental data (Hawarth [4]) shows good agreement of the numerical results.

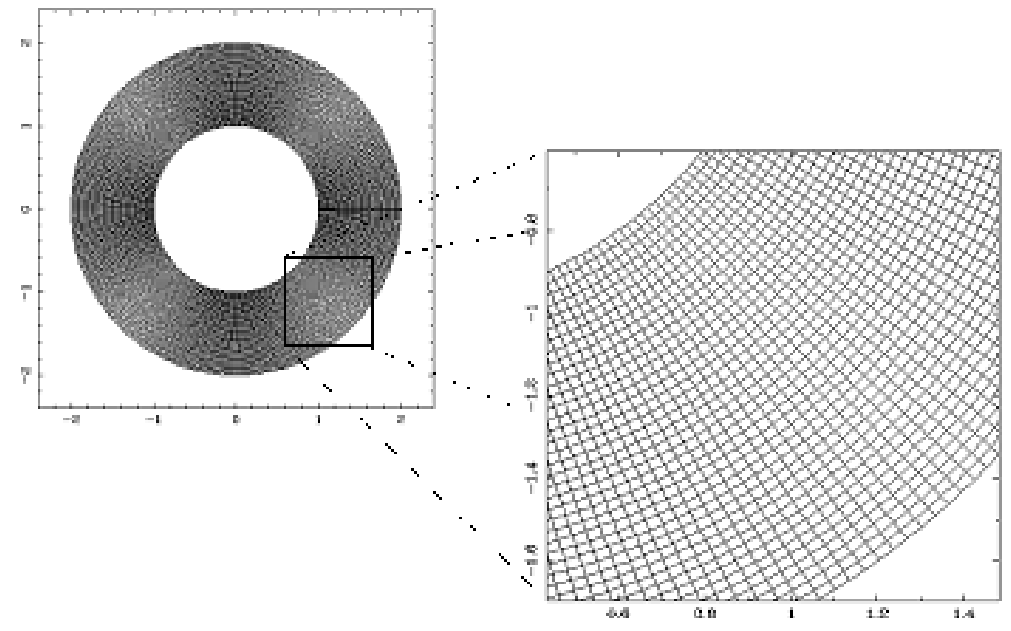

Fig. 4 Grid for concentric cylinders, $31 \times 61$ points

Following, Fig. 5 shows the isotherms for natural convection flow for $R_{a}=$ $10^{5}$ and $P_{r}=0.71$ for cylinder excentricity 0.8. Good agreement is observed when comparing these results with the ones presented by Ming-I et al., [7]. The isotherms are symmetric with respect to the vertical axis, and the heat tends to make the temperature uniform for the whole cavity. 


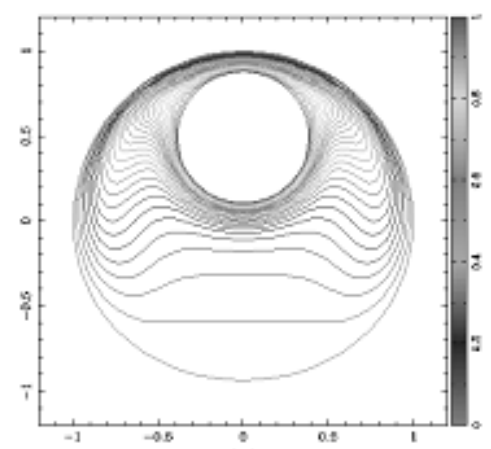

(a)

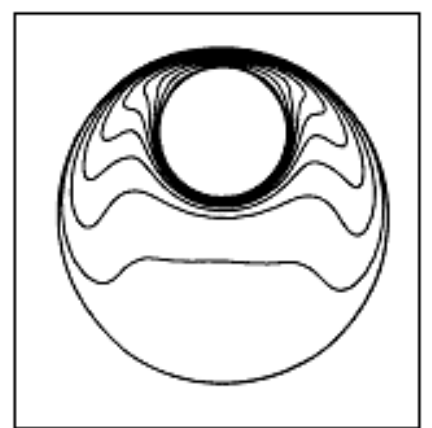

(b)

Fig. 5 Comparison of isotherms: (a) present work (b) Ming-I et al. [7];

$$
R_{e}=0, R_{a}=10^{5}
$$

The corresponding streamlines are presented in Fig. 6. They compare very well with the ones found by Ming-I et. al. [7], showing that the developed code is able to well represent the flow between excentric cylinders for a variety of non dimensional numbers mentioned before.

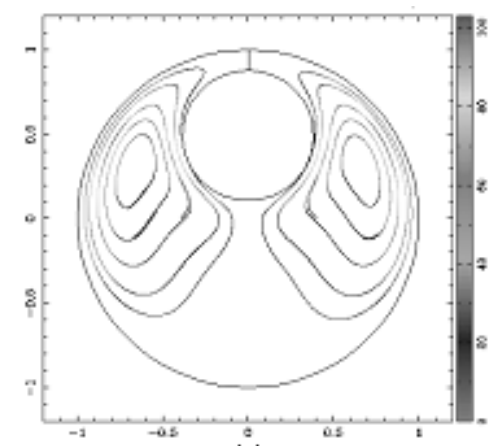

(a)

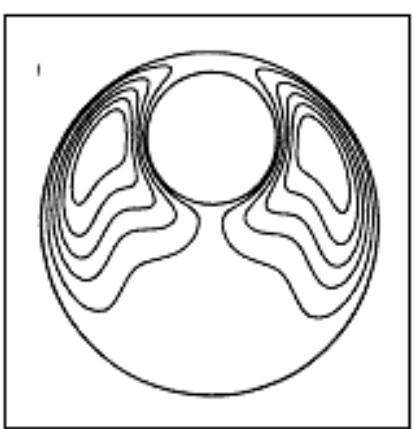

(b)

Fig. 6 Comparison of streamlines: (a) present work (b) Ming-I et al. [7];

$$
R_{e}=0, R_{a}=10^{5}
$$

Fig. 7 presents the flow for internal cylinder in movement for $R_{e}=378$ and $R_{a}$ $=10^{5}$. In this new situation the flow is not symmetric. Temperature distribution is also affected, as can be seen in Fig. 8, where centrifugal forces tend to produce a mixed convection (natural plus forced convection) flow, which is now more complex.

There appear small differences between the results of the present work and the ones obtained by Ming-I et al. [7], the latter using the finite volume method, whose results were compared with experimental ones.

Finally, numerical results for non-newtonian flows are presented and compared. The computational grid employed consists of $31 \mathrm{x} 61$ points. Mesh concentration is necessary mainly near walls using streching factor of order 1.1 (from line to line). Although this is a coarse grid, good temperature distribution was obtained. Fig. 
9 shows the isotherms obtained for laminar flow $\left(R_{e}=1, P_{r}=5.71\right.$ and $\left.D_{e}=0.01\right)$, between eccentric cylinders with eccentricity 0.8. The ratio between internal and external radius is 1.6 and the angular velocity is equal to 1.

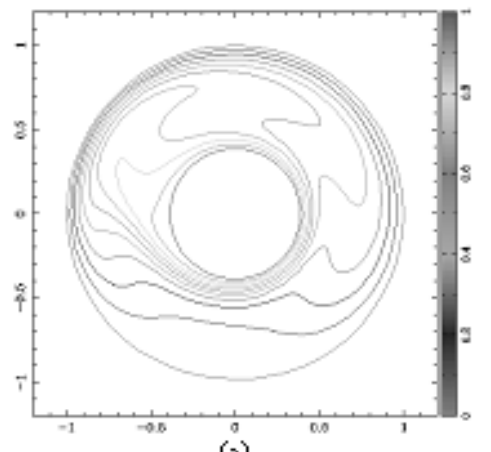

(a)

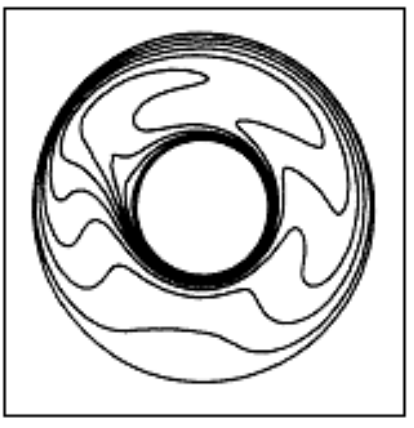

(b)

Fig. 7 Comparison of isotherms: (a) present work (b) Ming-I et al. [7];

$$
R_{e}=378 ., R_{a}=10^{5}
$$

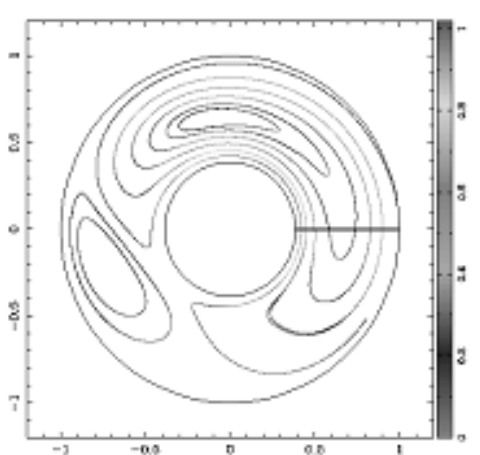

(a)

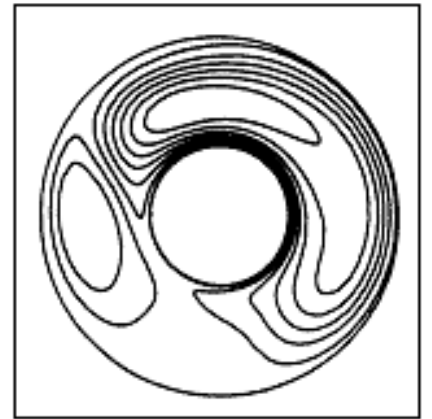

(b)

Fig. 8 Comparison of streamlines: (a) present work (b) Ming-I et al. [7]; $R_{e}=378, R_{a}=10^{5}$

Comparison of these results with the ones presented by Li e Davies [5] showed good agreement. The position of central isolines is approximatly the same, and the others have the same behaviour. 


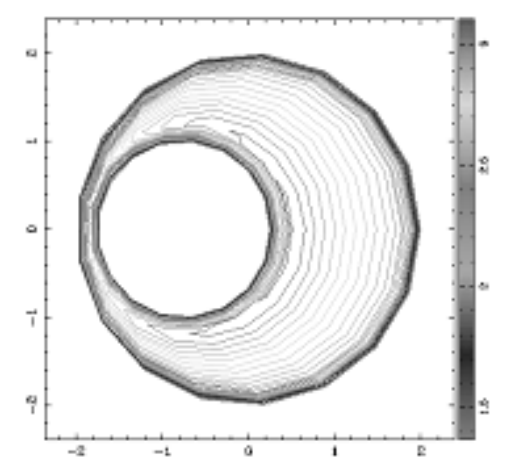

(a)

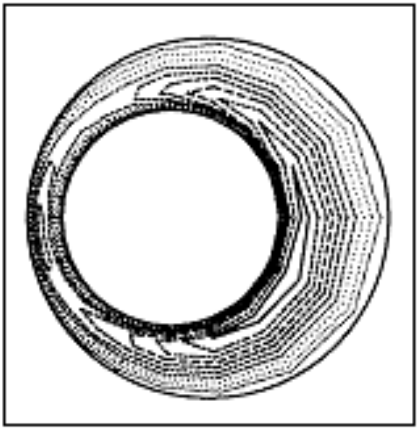

(b)

Fig. 9 Comparison of isotherms: (a) present work (b) Li e Davies [5], $R_{e}=1$ e $D_{e}=0.01$

\section{Conclusions}

Numerical tests have shown that the numerical method based on the finite differences spatial discretization and Runge-Kutta time-stepping scheme can be used to solve the flow between moving surfaces and the accuracy of the code has been compared through comparisons with analytical, experimental data found in the literature. The approach is obviously vectorizable and paralelizable.

Special care has been taken on the treatment of non newtonian terms (tensions), and a very good method capable of well represent newtonian as well as non newtonian flow behaviours was obtained. It was shown that the method works well with or without temperature variations, rotating or not, newtonian or non newtonian flows for cartesian as well as generalized geometries. Besides, the code structure was very simple allowing a performance around 1 Gigaflops at CRAY T-94 from CESUP-UFRGS, which is almost five times that of the common commercial codes used at that computer.

In conclusion, the numerical results obtained here indicate that there are many interesting physical phenomena associated with laminated flows between moving surfaces. This understanding is essential if real progress is to be done for more complex problems involving (non) newtonian flows whose applications for moving surfaces are obvious.

\section{Acknowledgement}

Authors would like to acknowledge that this work was developed employing CRAY T94 of CESUP - UFRGS.

Resumo. O objetivo do presente trabalho é a simulação numérica do fluxo entre superfícies móveis. O método baseia-se no processo de integração temporal de Runge-Kutta multiestágios com discretização espacial em diferenças centrais (segunda ordem). Testes são feitos para placas paralelas e superfícies cilíndricas 
móveis (mancais) para fluidos newtonianos e não-newtonianos, com e sem variações de temperatura e os resultados obtidos comparam adequadamente com resultados analíticos, numéricos e experimentais encontrados na literatura.

\section{References}

[1] D.A. Anderson, J.C. Tannehil and R.H. Pletcher, "Computational Fluid Mechanics and Heat Transfer", McGraw-Hill, New York, 1982.

[2] A. Bejan, "Convection Heat Transfer", John Wiley \& Sons, Inc., New York, 1984.

[3] M.J. Crochet, R.A. Davies and K. Walters, "Numerical Simulation of nonNewtonian Flow", Elsevier Science Publishers, Netherlands, 1984.

[4] L. Hawarth, "Modern Developments in Fluid Dynamics", Clarendon Press, 3rd edition, Oxford, 1964.

[5] X.K. Li and A.R. Davies, Thermal boundary condicitions for nonisothermal viscoelastic flow between eccentrically rotating cylinders, in "Developments in Non-Newtonian flows", ASME. FED-Vol. 206/AMD-Vol. 191, pp. 31-50, 1994.

[6] C.R. Maliska, "Transferência de Calor e Mecânica dos Fluidos Computacional", Editora Livro Técnico e Científico, Rio de Janeiro, 1995.

[7] C.H. Ming-I and Y-H. Hsu, Computation of buoyancy-driven flow in an eccentric centrifugal annulus with a non-orthogonal collocated finite volume algorithm, International Journal for Numerical Methods in Fluids 26 (1998), 323-343.

[8] H. Schlichting, "Boundary Layer Theory", McGraw-Hill, New York, 1968.

[9] J.A. Tichy, Non Newtonian lubrification with the convected Maxwell model, Journal of Tribology 118 (1996), 344-346.

[10] J.H. Wendt, "Computational Fluid Dynamics", Springer-Verlag, Berlin, 1996. 